TRANSACTIONS OF THE

AMERICAN MATHEMATICAL SOCIETY

Volume 363, Number 3, March 2011, Pages 1311-1323

S 0002-9947(2010)05036-6

Article electronically published on October 15, 2010

\title{
DUALITY IN SPACES OF FINITE LINEAR COMBINATIONS OF ATOMS
}

\author{
FULVIO RICCI AND JOAN VERDERA
}

\begin{abstract}
In this paper we describe the dual and the completion of the space of finite linear combinations of $(p, \infty)$-atoms, $0<p \leq 1$. As an application, we show an extension result for operators uniformly bounded on $(p, \infty)$-atoms, $0<p<1$, whose analogue for $p=1$ is known to be false. Let $0<p<1$ and let $T$ be a linear operator defined on the space of finite linear combinations of $(p, \infty)$-atoms, $0<p<1$, which takes values in a Banach space $B$. If $T$ is uniformly bounded on $(p, \infty)$-atoms, then $T$ extends to a bounded operator from $H^{p}\left(\mathbb{R}^{n}\right)$ into $B$.
\end{abstract}

\section{INTRODUCTION}

For each $0<p \leq 1$ consider the space $F^{p}$ of finite linear combinations of $(p, \infty)$ atoms, endowed with its natural norm (or quasi-norm for $p<1$ )

$$
\|f\|_{F^{p}}=\inf \left\{\left(\sum_{j}{ }^{\prime}\left|\lambda_{j}\right|^{p}\right)^{\frac{1}{p}}: f=\sum_{j}{ }^{\prime} \lambda_{j} a_{j}, a_{j} \text { a }(p, \infty) \text {-atom, } \lambda_{j} \in \mathbb{C}\right\},
$$

where $\sum^{\prime}$ denotes a finite sum. Recall that $a$ is a $(p, \infty)$-atom if $a$ is a measurable function supported on a ball $B$, satisfying the cancellation condition

$$
\int a(x) x^{\alpha} d x=0, \quad|\alpha| \leq n\left(\frac{1}{p}-1\right),
$$

and the size condition

$$
|a| \leq \frac{1}{|B|^{\frac{1}{p}}} .
$$

The space $F^{p}$ is clearly contained in $H^{p}=H^{p}\left(\mathbb{R}^{n}\right)$, the standard real Hardy space on $\mathbb{R}^{n}$. The elements of $H^{p}$ are the distributions that admit an atomic decomposition, $f=\sum_{j=1}^{\infty} \lambda_{j} a_{j}$, converging in the sense of distributions, for some $(p, \infty)$-atoms $a_{j}$ and scalars $\lambda_{j}$ with $\sum_{j=1}^{\infty}\left|\lambda_{j}\right|^{p}<\infty$ (for $p=1, H^{1} \subset L^{1}$ and atomic sums converge in the $L^{1}$-norm). In [MTW Meyer, Taibleson and Weiss observed that the $F^{p}$-norm is not comparable to the $H^{p}$-norm on $F^{p}$. Recently, it was shown in B that the Meyer-Taibleson-Weiss result leads to the following conclusion in the case $p=1$ : there exists a bounded linear functional on $F^{1}$ which does not extend to a bounded linear functional on $H^{1}$. In other words, there is a linear operator which is uniformly bounded on $(1, \infty)$-atoms but does not extend to a bounded linear operator on $H^{1}$.

Received by the editors October 23, 2008.

2010 Mathematics Subject Classification. Primary 42B30; Secondary 46J99.

(C)2010 American Mathematical Society 
In this paper we describe the structure of the completion $\widetilde{F^{p}}$ of $F^{p}, 0<p \leq 1$, and of its dual space. We show in particular that, when $p<1, F^{p}$ and $H^{p}$ have the same dual, and therefore no example like the one in $[\mathrm{B}$. can be exhibited for $p<1$. An immediate consequence of this is that if $0<p<1$ and the linear operator

$$
T: F^{p} \rightarrow B,
$$

maps $F^{p}$ into a Banach space $B$ satisfying the inequality

$$
\|T(a)\|_{B} \leq C,
$$

for some positive constant $C$ and all $(p, \infty)$-atoms, then $T$ extends to a bounded linear operator from $H^{p}$ into $B$. The argument proceeds by duality as follows. Take any $u$ in the dual $B^{*}$ of $B$. Since $u \circ T \in\left(F^{p}\right)^{*}=\left(H^{p}\right)^{*}$,

$$
|u(T(f))| \leq C\|u\|\|f\|_{H^{p}},
$$

and so, by the dual expression of the norm in a Banach space,

$$
\|T(f)\|_{B} \leq C\|f\|_{H^{p}} .
$$

We prove the following facts about $\widetilde{F^{p}}, 0<p \leq 1$.

(i) The closed subspace $\widetilde{F^{p}, c}$ of $\widetilde{F^{p}}$ spanned by the continuous $(p, \infty)$-atoms is isomorphic to $H^{p}$ as a Banach space, and $\widetilde{F^{p}}$ splits as the direct sum of $\widetilde{F^{p, c}}$ and a non-trivial complementary closed subspace $N^{p}$.

(ii) Every element $\xi$ of $\widetilde{F^{p}}$ admits an atomic decomposition

$$
\xi=\sum_{j=1}^{\infty} \lambda_{j} a_{j},
$$

for $(p, \infty)$-atoms $a_{j}$ and scalars $\lambda_{j}$ with $\sum_{j=1}^{\infty}\left|\lambda_{j}\right|^{p}<\infty$. Moreover, the $\widetilde{F^{p}}$-norm of $\xi$ is equivalent to its atomic norm

$$
\inf \left\{\left(\sum_{j=1}^{\infty}\left|\lambda_{j}\right|^{p}\right)^{\frac{1}{p}}: \sum_{j=1}^{\infty} \lambda_{j} a_{j}=\xi \text { in } \widetilde{F^{p}}\right\} .
$$

(iii) If an atomic sum $\sum_{j=1}^{\infty} \lambda_{j} a_{j}$, with $\lambda_{j}$ and $a_{j}$ as above, converges to 0 in $\widetilde{F^{p}}$, it also converges to 0 in $H^{p}$, but not vice versa. In fact, $N^{p}$ consists of those elements of $\widetilde{F^{p}}$ that are represented by atomic sums converging to 0 in $H^{p}$.

In other words, $H^{p}$ and $\widetilde{F^{p}}$ are both quotients of the space of "formal series" of $(p, \infty)$-atoms with $\ell^{p}$ coefficients, but the equivalence relation defining $\widetilde{F^{p}}$ is finer than that defining $H^{p}$.

So, the reason why $\left(F^{1}\right)^{*}$ is strictly larger than $\left(H^{1}\right)^{*}$ is that it is the direct sum of $\left(\widetilde{F^{1, c}}\right)^{*}=\left(H^{1}\right)^{*}$ and $\left(N^{1}\right)^{*}$. Notice that $\left(N^{1}\right)^{*}$ is non-trivial, as the dual of the non-trivial Banach space $N^{1}$. On the other hand, it turns out that $\left(N^{p}\right)^{*}$ is trivial for $p<1$.

To describe our results we need to introduce some notation and recall some basic classical facts in the theory of Banach algebras (see Section 3 for details).

Denote by $L_{0}^{\infty}\left(\mathbb{R}^{n}\right)$ the space of bounded measurable functions on $\mathbb{R}^{n}$ vanishing at infinity. Then $L_{0}^{\infty}\left(\mathbb{R}^{n}\right)$ is a commutative $C^{*}$-algebra without unit, and its maximal ideal space is a locally compact, non-compact space, which we call $\widehat{\mathbb{R}^{n}}$ (cf. F]). 
By the Gelfand-Naimark theorem, the Gelfand transform $f \rightarrow \hat{f}$ establishes an isometric isomorphism between $L_{0}^{\infty}\left(\mathbb{R}^{n}\right)$ and the algebra $C_{0}\left(\widehat{\mathbb{R}^{n}}\right)$ of all continuous functions on $\widehat{\mathbb{R}^{n}}$ vanishing at $\infty$. On the other hand, $C_{0}\left(\mathbb{R}^{n}\right)$ is a closed subalgebra of $L_{0}^{\infty}\left(\mathbb{R}^{n}\right)$, and its maximal ideal space is $\mathbb{R}^{n}$. This embedding induces a continuous projection $\pi$ from $\widehat{\mathbb{R}^{n}}$ onto $\mathbb{R}^{n}$. Clearly, if $f \in C_{0}\left(\mathbb{R}^{n}\right)$, then $\hat{f}=f \circ \pi$.

In a similar way, given any ball $B$ in $\mathbb{R}^{n}$, the maximal ideal space of $L^{\infty}(B)$ is a compact space $\widehat{B}$, endowed with a projection $\pi_{B}$ onto $\bar{B}$ induced by the inclusion of $C(\bar{B})$ in $L^{\infty}(B)$. Moreover, $L^{\infty}(B) \cong C(\widehat{B})$, again by the Gelfand-Naimark theorem.

The restriction map $f \mapsto f_{\left.\right|_{B}}$ from $L_{0}^{\infty}\left(\mathbb{R}^{n}\right)$ to $L^{\infty}(B)$ induces a natural embedding $\iota_{B}: \widehat{B} \rightarrow \widehat{\mathbb{R}^{n}}$, which is compatible with the projections $\pi$ and $\pi_{B}$, in the sense that

$$
\pi_{B}=\pi \circ \iota_{B}
$$

Similar embeddings $\iota_{B, B^{\prime}}: \widehat{B^{\prime}} \rightarrow \widehat{B}$ exist for pairs of balls $B, B^{\prime}$ with $B^{\prime} \subset B$, with the same compatibility with respect to the corresponding projections.

Denote by $m$ the Lebesgue measure on $\mathbb{R}^{n}$. The continuous linear functional $f \mapsto \int f d m$ on $L^{\infty}(B)$ is represented by a positive Borel measure $\widehat{m}_{B}$ on $\widehat{B}$, that is,

$$
\int f d m=\int \hat{f} d \widehat{m}_{B}, \quad f \in L^{\infty}(B) .
$$

If $B$ is contained in a second ball $B^{\prime}$, then the restriction of $\widehat{m}_{B^{\prime}}$ to $\widehat{B}$ is precisely $\widehat{m}_{B}$ and thus we can define a positive Borel measure $\widehat{m}$ globally on $\widehat{\mathbb{R}^{n}}$ by requiring that its restriction to $\widehat{B}$ be $\widehat{m}_{B}$ for each ball $B$.

We can now state our main result.

Theorem. (A) Let $\ell$ be a bounded linear functional on $F^{1}$. Then there exist a function $b \in B M O\left(\mathbb{R}^{n}\right)$ and a Radon measure $\mu$ on $\widehat{\mathbb{R}^{n}}$, singular with respect to $\widehat{m}$, satisfying

$$
|\mu|(\widehat{B}) \leq C m(B), \quad \text { for each ball } \quad B
$$

such that

$$
\ell(f)=\int f b d m+\int \hat{f} d \mu, \quad f \in F^{1} .
$$

Conversely, if $b$ and $\mu$ are as above, then the identity (15) defines a bounded linear functional on $F^{1}$ and

$$
\|\ell\|_{\left(F^{1}\right)^{*}} \cong\|b\|_{B M O}+\sup _{B} \frac{|\mu|(\widehat{B})}{m(B)} .
$$

(B) Each bounded linear functional on $F^{p}, 0<p<1$, extends uniquely to a bounded linear functional on $H^{p}\left(\mathbb{R}^{n}\right)$. Thus $\left(F^{p}\right)^{*}=H^{p}\left(\mathbb{R}^{n}\right)^{*}, 0<p<1$.

It is clear that relation (5) determines the function $b$ and the measure $\mu$ uniquely. Therefore $\left(F^{1}\right)^{*}$ differs from $\left(H^{1}\right)^{*}=B M O$ by the presence of the complementary subspace $S$ of singular measures satisfying (4). We will show that $S$ is non-trivial; in fact, the Meyer, Taibleson and Weiss argument may be interpreted as the construction of a non-zero measure in $S$. The decomposition of $\left(F^{1}\right)^{*}$ as $B M O \oplus S$ 
is the dual counterpart of the decomposition of $\widetilde{F^{1}}$ as $\widetilde{F^{1, c}} \oplus N^{1}$, although $S$ and $B M O$ do not coincide with the annihilators of $\widetilde{F^{1, c}}$ and $N^{1}$, respectively.

The nature of the elements of $N^{p}$, including $p=1$, is somehow mysterious. It is not clear at all to us if they can be represented by concrete analytic objects.

Section 2 contains the discussion of the completion of $F^{p}$ and a constructive argument which proves the non-triviality of $N^{p}$. In Section 3 we prove the Theorem. We also give an example of a non-zero singular measure satisfying (4).

We remark here that a variation of the main argument in the proof of the Theorem provides an alternative proof of some results in [MSV and [YZ) on the equivalence of the finite and infinite atomic norms of $(1, q)$-atoms, $q<\infty$, and on extension of bounded operators defined on finite linear combinations of $(p, q)$-atoms with $1<q<\infty$.

\section{The COMPletion of $F^{p}$}

Let $F^{p, c}$ stand for the subspace of $H^{p}$ consisting of finite linear combinations of continuous $(p, \infty)$ atoms. A surprising recent result in [MSV] states that the $H^{p}$ and the $F^{p}$ norms are equivalent on $F^{p, c}, 0<p \leq 1$. Indeed, the result is proved in [MSV] only for $p=1$, but, as suggested in Remark 3.2 there, the same argument extends to the case $0<p<1$. A complete proof may be found in MSV2, section $3]$.

More precisely, we can quote Lemma 3.1 and Remark 3.2 in [MSV] as follows.

Lemma 1. The following norms are equivalent on $F^{p, c}$ :

(a) the $H^{p}$-norm;

(b) the $F^{p}$-norm (11);

(c) the $F^{p, c}$-norm

$\|f\|_{F^{p, c}}=\inf \left\{\sum_{j}^{\prime}\left|\lambda_{j}\right|: f=\sum_{j}{ }^{\prime} \lambda_{j} a_{j}, a_{j}\right.$ a continuous $(p, \infty)$-atom, $\left.\lambda_{j} \in \mathbb{C}\right\}$.

Since $F^{p, c}$ is dense in $H^{p}$, the natural inclusion of $F^{p, c}$ in $F^{p}$ extends uniquely to a continuous linear operator $T$ from $H^{p}$ to $\widetilde{F^{p}}$. By Lemma 1, $T$ maps $H^{p}$ isomorphically onto the closure $\widetilde{F^{p}, c}$ of $F^{p, c}$ in $\widetilde{F^{p}}$. Notice that, again by Lemma 1, $\widetilde{F^{p, c}}$ is the completion of $F^{p, c}$ endowed either with the norm $\|\cdot\|_{F^{p, c}}$ or with the norm inherited from $F^{p}$.

On the other hand, the inclusion of $F^{p}$ (endowed with its natural norm) into $H^{p}$ is continuous, and it extends to a continuous linear operator $U$ from $\widetilde{F^{p}}$ to $H^{p}$. We then have the diagram

$$
H^{p} \stackrel{T}{\longrightarrow} \widetilde{F^{p}} \stackrel{U}{\longrightarrow} H^{p}
$$

with $U \circ T$ being the identity map. In particular, $U$ is surjective. Set $P=T \circ U$, so that $P$ is a projection, that is, $P^{2}=P$. The kernel of $P$ is the kernel of $U$, which we denote by $N^{p}$, and the kernel of $I-P$ is $T\left(H^{p}\right)=\widetilde{F^{p}, c}$. Hence we get the topological direct sum decomposition

$$
\widetilde{F^{p}}=\widetilde{F^{p, c}} \oplus N^{p} .
$$

Notice that $N^{p}$ is non-trivial, since otherwise the $H^{p}$ and the $F^{p}$ norms would be comparable on $F^{p}$.

To better understand the space $\widetilde{F^{p}}$ we now prove the following. 
Proposition. Given any sequence of $(p, \infty)$-atoms $a_{j}$ and any $\ell^{p}$-sequence of scalars $\lambda_{j}$, the series $\sum_{j=1}^{\infty} \lambda_{j} a_{j}$ converges in $\widetilde{F^{p}}$ to an element $\xi$ such that $\|\xi\|_{\widetilde{F^{p}}}^{p} \leq$ $\sum_{j=1}^{\infty}\left|\lambda_{j}\right|^{p}$. Conversely, each $\xi \in \widetilde{F^{p}}$ can be written as

$$
\xi=\sum_{j=1}^{\infty} \lambda_{j} a_{j}
$$

where each $a_{j}$ is a $(p, \infty)$-atom and the sum is convergent in $\widetilde{F^{p}}$. Moreover,

$$
\|\xi\|_{\widetilde{F^{p}}}^{p}=\inf \left\{\sum_{j=1}^{\infty}\left|\lambda_{j}\right|^{p}\right\}
$$

where the infimum is taken over all decompositions (6) of $\xi$.

Proof. Let $\xi$ be an element of $\widetilde{F^{p}}$. To prove (6), express $\xi$ as the limit in $\widetilde{F^{p}}$ of a sequence $S_{k}$ of elements of $F^{p}$. Given $\epsilon>0$, we may assume that $\left\|S_{1}\right\|_{F^{p}}^{p}<$ $(1+\epsilon)\|\xi\|_{\widetilde{F^{p}}}^{p}$ and that $\left\|S_{k}-S_{k+1}\right\|_{F^{p}}^{p}<\epsilon^{k}\|\xi\|_{\widetilde{F^{p}}}^{p}$. Thus

$$
\xi=\lim _{k \rightarrow \infty} S_{1}+\left(S_{2}-S_{1}\right)+\cdots+\left(S_{k}-S_{k-1}\right) .
$$

Set

$$
S_{1}=\sum_{j=1}^{N_{1}} \lambda_{j} a_{j},
$$

where the above expression has been chosen so that

$$
\sum_{j=1}^{N_{1}}\left|\lambda_{j}\right|^{p}<(1+\epsilon)\|\xi\|_{\widetilde{F^{p}}}^{p} .
$$

Similarly, set

$$
S_{\ell}-S_{\ell-1}=\sum_{j=N_{\ell-1}+1}^{N_{\ell}} \lambda_{j} a_{j}, \quad \ell \geq 2,
$$

with

$$
\sum_{j=N_{\ell-1}+1}^{N_{\ell}}\left|\lambda_{j}\right|^{p}<\epsilon^{\ell}\|\xi\|_{\widetilde{F^{p}}}^{p} .
$$

Then $\sum_{j=1}^{\infty}\left|\lambda_{j}\right|^{p}<(1-\epsilon)^{-1}\|\xi\|_{\widetilde{F^{p}}}^{p}$ and the partial sums $\xi_{m}=\sum_{j=1}^{m} \lambda_{j} a_{j}$ form a Cauchy sequence in $F^{p}$. This shows that (6) holds.

Notice also that, for each $\xi \in \widetilde{F^{p}}$, the inequality $\|\xi\|_{\widetilde{F^{p}}}^{p} \leq \inf \left\{\sum_{j=1}^{\infty}\left|\lambda_{j}\right|^{p}\right\}$, where the infimum is taken over all possible expressions (6), is due to the fact that $\|\cdot\|_{\widetilde{F^{p}}}^{p}$ satisfies the triangle inequality.

The atomic decomposition of elements of $\widetilde{F^{p}}$ given above provides an explicit description of the operator $U$.

Corollary. Let $\xi \in \widetilde{F^{p}}$ be represented by the sum (6). Then $U(\xi)$ is the sum of the same series in $H^{p}$. 
We end this section by providing a constructive proof of the non-triviality of $N^{p}$. Let us first describe the Meyer-Taibleson-Weiss construction as presented in [B]. Let $B$ denote the open ball centered at the origin with radius 1 . Take a sequence of open disjoint balls $B_{j}, j \geq 1$, such that $\bigcup_{j} B_{j}$ is dense in $B$. Notice that we may also choose the $B_{j}$ so that the Lebesgue measure of their union $\sum_{j \geq 1}\left|B_{j}\right|$ is as small as we wish. As shown in [B], for each $j$ there exists a (non-continuous) $(p, \infty)$-atom $a_{j}$ supported on $B_{j}$ with the property that $\left|a_{j}\right| \geq c\left|B_{j}\right|^{-\frac{1}{p}}$, where $c$ is a small positive constant depending only on $n$. Thus, setting

$$
f=\sum_{j \geq 1}\left|B_{j}\right|^{\frac{1}{p}} a_{j}
$$

we get $|f| \geq c$ on $\bigcup B_{j}$. From that it is not difficult to conclude (see $[\mathrm{B}$ ) that

$$
\|f\|_{F^{p}} \geq c|B|^{\frac{1}{p}} .
$$

On the other hand, we clearly have $\|f\|_{H^{p}}^{p} \leq \sum_{j \geq 1}\left|B_{j}\right|$, so that the ratio between $H^{p}$-norm and $F^{p}$-norm can be made as small as we wish.

We can now construct a sequence $\left\{f_{m}\right\}$ in $F^{p}$ satisfying

$$
\begin{aligned}
& \left\|f_{m}\right\|_{F^{p}}^{p} \geq c^{p}|B|, \\
& \left\|f_{m}-f_{m+1}\right\|_{F^{p}}^{p} \leq 2^{p} \frac{|B|}{2^{m}}, \\
& \left\|f_{m}\right\|_{H^{p}}^{p} \leq \frac{|B|}{2^{m}} .
\end{aligned}
$$

The first two conditions imply that $\left\{f_{m}\right\}$ has a non-zero limit $\xi \in \widetilde{F^{p}}$, whereas the third implies that $U f_{m}=f_{m}$ tends to 0 in $H^{p}$. Hence $\xi \in N^{p}$.

The functions $f_{m}$ have the form (8); precisely,

$$
f_{m}=\sum_{j \geq 1}\left|B_{j}^{m}\right|^{\frac{1}{p}} a_{j}^{m},
$$

where, for each $m,\left\{B_{j}^{m}\right\}_{j}$ is a disjoint family of balls contained in $B$ with dense union and small total measure, and each $a_{j}^{m}$ is a $(p, \infty)$-atom with $\left|a_{j}^{m}\right| \geq c\left|B_{j}^{m}\right|^{-\frac{1}{p}}$.

The first function $f_{1}$ can be any function as in (8) with, say, $\sum_{j \geq 1}\left|B_{j}^{1}\right|<|B| / 2$. We then construct inductively $f_{m+1}$ from $f_{m}$ as follows.

Take $N$ so large that $\sum_{j>N}\left|B_{j}^{m}\right|<(1 / 4) \sum_{j \geq 1}\left|B_{j}^{m}\right|$. Inside each $B_{j}^{m}, 1 \leq j \leq$ $N$, we take open disjoint balls $B_{j l}^{\prime}, l \geq 1$, such that $\bigcup_{l \geq 1} B_{j l}^{\prime}$ is dense in $B_{j}^{m}$ and $\sum_{l \geq 1}\left|B_{j l}^{\prime}\right|<\left|B_{j}^{m}\right| / 4$

Then

$$
\sum_{j=1}^{N} \sum_{l \geq 1}\left|B_{j l}^{\prime}\right|+\sum_{j>N}\left|B_{j}^{m}\right| \leq \frac{1}{2} \sum_{j \geq 1}\left|B_{j}^{m}\right| .
$$

Let $a_{j l}^{\prime}$ be a $(p, \infty)$-atom supported on $B_{j l}^{\prime}$ with $\left|a_{j l}^{\prime}\right| \geq c\left|B_{j l}^{\prime}\right|^{-\frac{1}{p}}$. Set

$$
f_{m+1}=\sum_{j=1}^{N} \sum_{l \geq 1}\left|B_{j l}^{\prime}\right|^{\frac{1}{p}} a_{j l}^{\prime}+\sum_{j>N}\left|B_{j}^{m}\right|^{\frac{1}{p}} a_{j}^{m} .
$$


Since $\left|f_{m+1}\right| \geq c$ on an open dense subset of $B,\left\|f_{m+1}\right\|_{F^{p}}^{p} \geq c^{p}|B|$. Moreover,

$$
f_{m}-f_{m+1}=\sum_{j=1}^{N}\left(\left|B_{j}^{m}\right|^{\frac{1}{p}} a_{j}^{m}-\sum_{l \geq 1}\left|B_{j l}^{\prime}\right|^{\frac{1}{p}} a_{j l}^{\prime}\right) .
$$

For each $j$, the function

$$
\left|B_{j}^{m}\right|^{\frac{1}{p}} a_{j}^{m}-\sum_{l \geq 1}\left|B_{j l}^{\prime}\right|^{\frac{1}{p}} a_{j l}^{\prime}
$$

is supported on $B_{j}^{m}$ and its absolute value is not greater than 2 . Hence

$$
\left\|f_{m}-f_{m+1}\right\|_{F^{p}}^{p} \leq \sum_{j=1}^{N} 2^{p}\left|B_{j}^{m}\right| .
$$

Now, we relabel the balls in such a way that

$$
\left\{B_{j}^{m+1}\right\}_{j \geq 1}=\left\{B_{j}^{m}\right\}_{j>N} \cup\left\{B_{j l}^{\prime}\right\}_{j \leq N, l \geq 1},
$$

and rename the atoms in $f_{m+1}$ as $a_{j}^{m+1}$ accordingly. Then, inductively from (10),

$$
\sum_{j \geq 1}\left|B_{j}^{m}\right| \leq 2^{-m}|B|
$$

for every $m$, and the required estimates can be easily verified.

\section{Proof of the Theorem}

We start by proving, for the reader's sake, a few statements made (explicitly or not) in the last part of the introduction concerning the Gelfand spectrum $\widehat{\mathbb{R}^{n}}$ and its projection $\pi$ on $\mathbb{R}^{n}$.

The first statement we want to prove is that $\pi$ is, in fact, well defined. Given $\phi$ in $\widehat{\mathbb{R}^{n}}$, i.e., a non-trivial multiplicative functional on $L_{0}^{\infty}\left(\mathbb{R}^{n}\right)$, it is clear that its restriction to $C_{0}\left(\mathbb{R}^{n}\right)$ is also multiplicative. We must show that this restriction is "evaluation" at some point $x=\pi(\phi)$ of $\mathbb{R}^{n}$, or, equivalently, that it is not identically zero.

Since $L_{0}^{\infty}\left(\mathbb{R}^{n}\right)$ is a $C^{*}$-algebra, it is symmetric, so that $\phi(\bar{f})=\overline{\phi(f)}$ for every $f$. Therefore, $f \geq 0$ implies that $\phi(f) \geq 0$, so that $\phi$ is monotonic on real-valued functions. If $\phi$ vanishes identically on $C_{0}\left(\mathbb{R}^{n}\right)$, it also vanishes on characteristic functions of compact sets. By linearity and continuity, this would be a contradiction.

The second statement is that the mapping $\pi$ is surjective. We know that to each $\phi \in \widehat{\mathbb{R}}^{n}$ we can associate a point $\pi(\phi)$ in $\mathbb{R}^{n}$. Given $y \in \mathbb{R}^{n}$, we can define a translate $\tau_{y} \phi \in \widehat{\mathbb{R}^{n}}$ by

$$
\tau_{y} \phi(f)=\phi(f(\cdot+y)) .
$$

It is quite clear that $\pi\left(\tau_{y} \phi\right)=\pi(\phi)+y$. Since $\widehat{\mathbb{R}^{n}}$ is non-empty, $\pi$ is surjective.

The last statement which remained unproved in the introduction is that $\widehat{\mathbb{R}^{n}}$ is the union of the $\widehat{B}$ over all balls $B$. This is a direct consequence of (ii) in the following lemma. 
Lemma 2. Let $B$ be an open ball in $\mathbb{R}^{n}$. Then

(i)

$$
\widehat{B}=\left\{\phi \in \widehat{\mathbb{R}^{n}}: \phi\left(\chi_{B}\right)=1\right\}=\operatorname{supp} \widehat{\chi_{B}},
$$

where $\widehat{f}$ stands for the Gelfand transform of $f \in L_{0}^{\infty}\left(\mathbb{R}^{n}\right)$.

$$
\pi^{-1}(B) \subset \widehat{B} \subset \pi^{-1}(\bar{B}) .
$$

Proof. To prove (12) notice that $\phi\left(\chi_{B}\right)$ is either 0 or 1 by the multiplicative property.

If $\phi\left(\chi_{B}\right)=1$, then $\phi(f)=\phi\left(f \chi_{B}\right), f \in L_{0}^{\infty}\left(\mathbb{R}^{n}\right)$, which means that $\phi$ factors through a character of $L^{\infty}(B)$. Thus $\phi \in \widehat{B}$. The argument can be reversed, so (12) is proved.

Assume now that for some $\phi \in \widehat{\mathbb{R}^{n}}$ we have $\pi(\phi) \in B$. Let $f$ be a continuous function on $\mathbb{R}^{n}$, with $f(\pi(\phi))=1$ and compact support contained in $B$. Then $f \chi_{B}=f$ and so

$$
1=\phi(f)=\phi(f) \phi\left(\chi_{B}\right)=\phi\left(\chi_{B}\right) .
$$

Then $\phi \in \widehat{B}$ because of (12).

If $\pi(\phi)$ is not in $\bar{B}$, then there is a continuous function $f$ on $\mathbb{R}^{n}$, with $f(\pi(\phi))=1$ and compact support in $\mathbb{R}^{n} \backslash \bar{B}$. Thus $f \chi_{B}=0$ and so $\phi\left(\chi_{B}\right)=0$, that is, $\phi$ is not in $\widehat{B}$.

We now turn to the proof of the Theorem. We begin by discussing the converse statement in part (A) of the Theorem. Obviously, given $b \in B M O$, the linear functional $f \mapsto \int f b d m$ is bounded on $\widetilde{F^{1}}$ with a norm controlled from above by the $B M O$-norm of $b$. On the other hand, restriction of the functional to $\widetilde{F^{1, c}}$ gives a control from below by the same $B M O$-norm.

We first remark that (12) clearly implies that, given $f \in L_{0}^{\infty}\left(\mathbb{R}^{n}\right)$, the support of $f$ is contained in $B$ if and only if the support of $\widehat{f}$ is contained in $\widehat{B}$.

Let $\mu$ be a Radon measure on $\widehat{\mathbb{R}^{n}}$ satisfying (4). For each $(1, \infty)$-atom $a$ supported on a ball $B$ one has

$$
\left|\int \widehat{a} d \mu\right| \leq\|a\|_{\infty}|\mu|(\widehat{B}) \leq \frac{|\mu|(\widehat{B})}{m(B)}<C .
$$

Hence $\mu$ determines a bounded linear functional on $\widetilde{F^{1}}$.

Assume now that $\ell$ is a bounded linear functional on $F^{1}$. Fix a ball $B$ and let $L_{0}^{\infty}(B)$ stand for the set of functions in $L^{\infty}(B)$ with zero integral. Given $f \in$ $L_{0}^{\infty}(B)$,

is a $(1, \infty)$-atom. Thus

$$
\frac{1}{m(B)} \frac{f}{\|f\|_{\infty}}
$$

$$
|\ell(f)| \leq\|\ell\|\|f\|_{\infty} m(B), \quad f \in L_{0}^{\infty}(B) .
$$

The restriction of $\ell$ to $L_{0}^{\infty}(B)$ extends to a bounded linear functional on $L^{\infty}(B)=$ $C(\widehat{B})$. Thus there exists a measure $\nu_{B}$ on $\widehat{B}$ such that

$$
\ell(f)=\int \widehat{f} d \nu_{B}, \quad f \in L_{0}^{\infty}(B) .
$$


If $f \in L^{\infty}(B)$, then clearly $\widehat{f}_{\widehat{B}}=f_{B}$, where $g_{E}$ stands for the mean of the function $g$ on the set $E$ with respect to the underlying measure ( $\widehat{m}$ or $m$ in the case at hand). Then

$$
\begin{aligned}
\ell\left(f-f_{B}\right) & =\int_{\widehat{B}}\left(\widehat{f}-\widehat{f}_{\widehat{B}}\right) d \nu_{B} \\
& =\int_{\widehat{B}}\left(\widehat{f}-\widehat{f}_{\widehat{B}}\right)\left(d \nu_{B}-\nu_{B}(\widehat{B}) \frac{d \widehat{m}}{\widehat{m}(\widehat{B})}\right) \\
& =\int_{\widehat{B}} \widehat{f}\left(d \nu_{B}-\frac{\nu_{B}(\widehat{B})}{\widehat{m}(\widehat{B})} d \widehat{m}\right),
\end{aligned}
$$

for each $f \in L^{\infty}(B)$. Therefore, if $\nu_{B}$ represents $\ell$ on $L_{0}^{\infty}(B)$, that is, if (14) holds, then $d \nu_{B}-\nu_{B}(\widehat{B}) \frac{\widehat{m}}{\widehat{m}(\widehat{B})}$ is uniquely determined.

Let $B_{N}$ stand for the open ball with center at the origin and radius $N, N=$ $1,2, \ldots$. Take any measure $\nu_{1}$ on $\widehat{B_{1}}$ that represents $\ell$ on $L_{0}^{\infty}\left(B_{1}\right)$. Every other such measure differs from $\nu_{1}$ by a constant multiple of $\chi_{\widehat{B_{1}}} \widehat{m}$. By the preceding remark applied to $B_{N}$ there exists a unique measure $\nu_{N}$ on $\widehat{B_{N}}$ which represents $\ell$ on $L_{0}^{\infty}\left(B_{N}\right)$ and $\nu_{N}\left(B_{1}\right)=\nu_{1}\left(B_{1}\right)$. Clearly $\nu_{N}$ restricted to $\widehat{B_{N-1}}$ is precisely $\nu_{N-1}$. Therefore we can define a measure $\nu$ on $\widehat{\mathbb{R}^{n}}$ by requiring that $\nu$ restricted to $\widehat{B_{N}}$ be $\nu_{N}$.

Given any ball $B$ take $N$ such that $B \subset B_{N}$. Since the restriction of $\nu$ to $\widehat{B_{N}}$ represents $\ell$ on $L_{0}^{\infty}\left(B_{N}\right)$, which contains $L_{0}^{\infty}(B)$, the restriction of $\nu$ to $\widehat{B}$ represents $\ell$ on $L_{0}^{\infty}(B)$ as well. By (15),

$$
\left|\int_{\widehat{B}} \widehat{f}\left(d \nu-\nu(\widehat{B}) \frac{d \widehat{m}}{\widehat{m}(\widehat{B})}\right)\right| \leq 2\|\ell\|\|f\|_{\infty} m(B), \quad f \in L^{\infty}(B)
$$

or

$$
\left\|\nu-\nu(\widehat{B}) \frac{\widehat{m}}{\widehat{m}(\widehat{B})}\right\|_{\widehat{B}} \leq 2\|\ell\| m(B) .
$$

Let us now consider the Radon-Nikodym decomposition of $\nu$

$$
\nu=g \widehat{m}+\mu,
$$

where $g \in L_{\text {loc }}^{1}(\widehat{m})$ and $\mu$ is singular with respect to $\widehat{m}$. By (16),

$$
|\mu|(\widehat{B}) \leq 2\|\ell\| m(B)
$$

and

$$
\int_{\widehat{B}}\left|g-g_{\widehat{B}}-\frac{\mu(\widehat{B})}{\widehat{m}(\widehat{B})}\right| d \widehat{m} \leq 2\|\ell\| m(B) .
$$

We are left with the task of finding the $B M O$-function $b$.

Combining (17) and (18) we readily get

$$
\int_{\widehat{B}}\left|g-g_{\widehat{B}}\right| d \widehat{m} \leq 4\|\ell\| m(B) .
$$

We need a lemma. 
Lemma 3. For each function $g \in L_{\text {loc }}^{1}(\widehat{m})$ there exists a unique function $f \in$ $L_{\text {loc }}^{1}(m)$ with the property that for each ball $B$,

$$
\int g \widehat{\varphi} d \widehat{m}=\int f \varphi d m, \quad \varphi \in L^{\infty}(B) .
$$

Such $f$ satisfies

$$
\int_{\widehat{B}}\left|g-g_{\widehat{B}}\right| d \widehat{m}=\int_{B}\left|f-f_{B}\right| d m
$$

for each ball $B$.

Once the lemma is proved we complete the proof of part (A) of the Theorem by just calling $b$ the function $f$ associated with $g$ in Lemma 3. Inequality (19) tells us that $b \in B M O\left(\mathbb{R}^{n}\right)$ and that its $B M O\left(\mathbb{R}^{n}\right)$ norm is not greater than $4\|\ell\|$.

Proof of Lemma 3. We will show that for each ball $B$ the Gelfand transform, which is an isometry between $L^{\infty}(B)$ and $C(\widehat{B})$, extends to an isometry between $L^{1}(B, m)$ and $L^{1}(\widehat{B}, \widehat{m})$. This immediately provides a further extension of the Gelfand transform to a topological isomorphism between $L_{\mathrm{loc}}^{1}(m)$ and $L_{\mathrm{loc}}^{1}(\widehat{m})$.

We begin by showing that, for each ball $B$ in $\mathbb{R}^{n}$ and every $f \geq 0$ in $L^{\infty}(B)$,

$$
\int_{\widehat{B}} \widehat{f} d \widehat{m}=\int_{B} f d m
$$

This follows from

$$
\begin{aligned}
\int_{B} f d m & =\sup _{\varphi} \int_{B} f \varphi d m \\
& =\sup _{\varphi} \int_{\widehat{B}} \widehat{f} \widehat{\varphi} d \widehat{m} \\
& =\int_{\widehat{B}} \widehat{f} d \widehat{m},
\end{aligned}
$$

where the supremum is taken on the closed unit ball of $L^{\infty}(B)$.

By linearity, (20) provides an extension of the Gelfand transform to a topological isomorphisms $f \rightarrow \widehat{f}$ of $L_{\mathrm{loc}}^{1}(m)$ onto $L_{\mathrm{loc}}^{1}(\widehat{m})$. Given $g \in L_{\mathrm{loc}}^{1}(\widehat{m})$ take $f \in L_{\mathrm{loc}}^{1}(m)$ with $g=\widehat{f}$. The first identity in the statement of Lemma 3 follows by approximating $f \in L^{1}(B, m)$ by functions in $L^{\infty}(B)$ and the second follows from (20).

Before proving part (B) of the Theorem we give an explicit example, modeled on the Meyer-Taibleson-Weiss argument, of a non-zero measure which is singular with respect to $\widehat{m}$ and satisfies (4).

Take an open set $U$ of $\mathbb{R}^{n}, U \subset B_{0}=\{x:|x| \leq 1\}$, such that $U$ is dense in $B_{0}$ and $m(U)<m\left(B_{0}\right)$. Then the compact set $E=B_{0} \backslash U$ has positive Lebesgue measure. Set $V=\pi^{-1}(U)$, so that $V \subset \widehat{B_{0}}$ by Lemma 1 . Then $U \subset \pi(\bar{V})$ and so $\pi(\bar{V})=B_{0}$, because $U$ is dense in $B_{0}$. Hence $\pi(\partial V)=E$. Now, the boundary of each open set in $\widehat{B_{0}}$ has zero $\widehat{m}$ measure $([\underline{\mathrm{R}}, \mathrm{p} .286])$. Therefore $\widehat{m}(\partial V)=0$ but $m(\pi(\partial V))=m(E)>0$. Identify $C(E)$ to the subspace $S$ of continuous functions on $\partial V$ of the form $f \circ \pi, f \in C(E)$. The bounded linear functional on $S$ defined 
by $f \rightarrow \int f d m$ extends by Hahn-Banach to a bounded linear functional on $C(\partial V)$ with the same norm. Thus there exists a positive measure $\mu$ on $\partial V$ such that

$$
\int(f \circ \pi) d \mu=\int f d m, \quad f \in C(E) .
$$

If $B$ is an open ball, then by Lemma 2 ,

$$
\mu(\widehat{B}) \leq \mu\left(\pi^{-1}(\bar{B})\right)=m(\bar{B} \cap E) \leq m(B),
$$

and condition (4) is satisfied.

Proof of part (B) of the Theorem. The argument is analogous to the proof of part (A), except for minor technical details. If $0<p<1$, then, as we will see, the singular measure $\mu$ vanishes and so we will conclude that $\left(F^{p}\right)^{*}=H^{p}\left(\mathbb{R}^{n}\right)^{*}$.

Let $\ell$ be a bounded linear functional on $F^{p}, 0<p<1$. Let $d$ be the integer part of $n\left(\frac{1}{p}-1\right)$. Given a ball $B$ let $L_{d}^{\infty}(B)$ stand for the set of functions $f \in L^{\infty}(B)$ such that

$$
\int f(x) x^{\alpha} d x=0, \quad|\alpha| \leq d
$$

For each $f \in L_{d}^{\infty}(B)$,

$$
\frac{1}{m(B)^{\frac{1}{p}}} \frac{f}{\|f\|_{\infty}}
$$

is a $(p, \infty)$-atom and so

$$
|\ell(f)| \leq\|\ell\|\|f\|_{\infty} m(B)^{\frac{1}{p}}, \quad f \in L_{d}^{\infty}(B) .
$$

For each $f \in L^{\infty}(B)$ let $P_{B}(f)$ be (the restriction to $B$ of) the unique polynomial of degree not greater than $d$ such that

$$
\int f(x) x^{\alpha} d x=\int_{B} P_{B}(f)(x) x^{\alpha} d x, \quad|\alpha| \leq d .
$$

Since $P_{B}(f)$ is the orthogonal projection (in $L^{2}(B)$ ) of $f$ into the subspace of polynomials of degree not greater than $d$,

$$
\left\|P_{B}(f)\right\|_{2} \leq\|f\|_{2} \leq\|f\|_{\infty}
$$

where the $L^{2}$ norms are taken with respect to the normalized Lebesgue measure on $B$. Now, we want to compare the norms $\|\cdot\|_{2}$ and $\|\cdot\|_{\infty}$ on the space $P_{d}(B)$ of restrictions to $B$ of polynomials of degree not greater than $d$. After appropriate translation and dilation we may assume that $B$ has center 0 and radius 1 . Since $P_{d}(B)$ is finite dimensional, there is a constant $C(d, n)$, depending only on $d$ and $n$, such that

$$
\|P\|_{\infty} \leq C(d, n)\|P\|_{2}, \quad P \in P_{d}(B),
$$

and so

$$
\left\|P_{B}(f)\right\|_{\infty} \leq C(d, n)\|f\|_{\infty}, \quad f \in L^{\infty}(B) .
$$

Therefore by (21),

$$
\left|\ell\left(f-P_{B}(f)\right)\right| \leq(1+C(d, n))\|\ell\|\|f\|_{\infty} m(B)^{\frac{1}{p}}, \quad f \in L^{\infty}(B) .
$$

By (21) there is a measure $\nu_{B}$ on $\widehat{B}$ such that

$$
\ell(f)=\int \widehat{f} d \nu_{B}, \quad f \in L_{d}^{\infty}(B) .
$$


Given a measure $\lambda$ on $\widehat{B}$ there is a unique polynomial $P_{B}(\lambda) \in P_{d}(B)$ such that

$$
\int_{\widehat{B}}(\pi(\phi))^{\alpha} d \lambda(\phi)=\int_{B} P_{B}(\lambda)(x) x^{\alpha} d x, \quad|\alpha| \leq d .
$$

Hence, for every polynomial $Q$ of degree $\leq d$,

$$
\int_{\widehat{B}} \widehat{Q} d \lambda=\int_{B} P_{B}(\lambda) Q d m=\int_{\widehat{B}} \widehat{P_{B}(\lambda)} \widehat{Q} d \widehat{m} .
$$

Therefore, by (23),

$$
\begin{aligned}
\ell\left(f-P_{B}(f)\right) & =\int_{\widehat{B}}\left(\widehat{f}-\widehat{P_{B}(f)}\right) d \nu_{B} \\
& =\int_{\widehat{B}}\left(\widehat{f}-\widehat{P_{B}(f)}\right)\left(d \nu_{B}-\widehat{P_{B}\left(\nu_{B}\right)} d \widehat{m}\right) \\
& \left.=\int_{\widehat{B}} \widehat{f}\left(d \nu_{B}-\widehat{P_{B}\left(\nu_{B}\right.}\right) d \widehat{m}\right),
\end{aligned}
$$

for each $f \in L^{\infty}(B)$. Hence the measure $\nu_{B}-\widehat{P_{B}\left(\nu_{B}\right)} \widehat{m}$ is determined by $\ell$.

As before, with $B_{N}$ denoting the ball of radius $N$ centered at the origin, we fix a measure $\nu_{1}$ on $\widehat{B_{1}}$ that represents $\ell$ on $L_{d}^{\infty}\left(B_{1}\right)$ and then take the unique measure $\nu_{N}$ on $\widehat{B_{N}}$ which represents $\ell$ on $L_{d}^{\infty}\left(B_{N}\right)$ and such that $P_{B_{1}}\left(\nu_{N}\right)=P_{B_{1}}\left(\nu_{1}\right)$. Then $\nu_{N}$ restricted to $B_{N-1}$ is $\nu_{N-1}$, so we can define a measure $\nu$ on $\widehat{\mathbb{R}^{n}}$ by requiring that $\nu$ restricted to $B_{N}$ be $\nu_{N}$.

Given any ball $B$, take $N$ such that $B \subset B_{N}$. Then the restriction of $\nu$ to $L_{d}^{\infty}(B)$ is $\ell$, so, by (22) and (24),

$$
\left|\int_{\widehat{B}} \widehat{f}\left(d \nu-\widehat{P_{B}(\nu)} d \widehat{m}\right)\right| \leq C\|f\|_{\infty} m(B)^{\frac{1}{p}}, \quad f \in L^{\infty}(B) .
$$

Hence

$$
\left|\nu-\widehat{P_{B}(\nu)} \widehat{m}\right|(\widehat{B}) \leq C m(B)^{\frac{1}{p}} .
$$

Now consider the Radon-Nikodym decomposition of $\nu$,

$$
\nu=g \widehat{m}+\mu,
$$

with $\mu$ singular with respect to $\widehat{m}$. We get, by (25) and Lemma 2

$$
|\mu|\left(\pi^{-1}(B)\right) \leq|\mu|(\widehat{B}) \leq C m(B)^{\frac{1}{p}},
$$

for each open ball $B$. Since $0<p<1$, we readily conclude that $\mu=0$. Indeed, let $r$ be the radius of $B$. Covering $B$ by $A_{n} k^{n}$ balls of radius $r / k$, we see that the constant $C$ in the right-hand side of the above inequality can be replaced by $C A_{n} k^{n\left(1-\frac{1}{p}\right)}$. Letting $k$ tend to $\infty$, we obtain the conclusion.

Now take $f \in L_{\text {loc }}^{1}\left(\mathbb{R}^{n}\right)$ with $g=\widehat{f}$. Then

$$
\int_{B}\left|f-P_{B}(f)\right| d m \leq C m(B)^{\frac{1}{p}},
$$

which is precisely the condition that guarantees that $f$ determines a bounded linear functional on $H^{p}\left(\mathbb{R}^{n}\right)$ ( $[\mathrm{TW}]$ ). Thus $\ell$ is a bounded linear functional on $H^{p}\left(\mathbb{R}^{n}\right)$ and the proof is complete. 


\section{ACKNOWLEDGMENTS}

The second author wishes to express his gratitude for the warm hospitality of the "Centro di Ricerca Matematica E. De Giorgi" during the spring of 2008. The second author was partially supported by grants 2005SGR00774 ("Generalitat de Catalunya"), MTM2007-60062 and a "Ayuda" from "Ministerio de Educación y Ciencia".

\section{REFERENCES}

[B] M. Bownik, Boundedness of operators on Hardy spaces via atomic decompositions, Proc. Amer. Math. Soc. 133(2005), 3535-3542. MR2163588 (2006d:42028)

[F] G. Folland, A Course in Abstract Harmonic Analysis, Studies in Advanced Mathematics, CRC Press, 1995. MR1397028 (98c:43001)

[MSV] S. Meda, P. Sjogren and M. Vallarino, On the $H^{1}-L^{1}$ boundedness of operators, Proc. Amer. Math. Soc. 136 (8)(2008), 2921-2931. MR2399059 (2009b:42025)

[MSV2] S. Meda, P. Sjogren and M. Vallarino, Atomic decompositions and operators on Hardy spaces, Rev. Un. Mat. Argentina 50 (2009), 15-22. MR.2656522

[MTW] Y. Meyer, M. H. Taibleson and G. Weiss, Some functional analytic properties of the spaces $B_{q}$ generated by blocks, Indiana U. Math. J. 34 (3)(1985), 493-515. MR794574 (87c:46036)

[R] W. Rudin, Functional Analysis, International Series in Pure and Applied Mathematics, McGraw-Hill, Inc., New York, 1991, Second edition. MR.1157815 (92k:46001)

[TW] M. H. Taibleson and G. Weiss, The molecular characterization of certain Hardy spaces, Astérisque 77, Societe Mathematique de France, (1980). MR604370 (83g:42012)

[YZ] D. Yang and Y. Zhou, A boundedness criterion via atoms for linear operators in Hardy spaces, Constructive Approximation 29 (2009), no. 2, 207-218. MR2481589 (2010e:42021)

Reparto di Matematica, Scuola Normale Superiore di Pisa, Piazza dei Cavalieri 7 , 56126 Pisa, ItAlia

E-mail address: fricci@sns.it

Departament de Matemàtiques, Universitat Autònoma de Barcelona, 08193 Bellaterra, Barcelona, Catalonia

E-mail address: jvm@mat.uab.cat 